\title{
STRUCTURE AND REPRESENTATIONS OF THE QUANTUM GENERAL LINEAR SUPERGROUP
}

\author{
R. B. ZHANG \\ Department of Pure Mathematics \\ University of Adelaide \\ Adelaide, Australia
}

\begin{abstract}
The structure and representations of the quantum general linear supergroup $G L_{q}(m \mid n)$ are studied systematically by investigating the Hopf superalgebra $G_{q}$ of its representative functions. $G_{q}$ is factorized into $G_{q}^{\pi} G_{q}^{\bar{\pi}}$, and a Peter - Weyl basis is constructed for each factor. Parabolic induction for the quantum supergroup is developed. The underlying geometry of induced representations is discussed, and an analog of Frobenius reciprocity is obtained. A quantum Borel - Weil theorem is proven for the covariant and contravariant tensorial irreps, and explicit realizations are given for classes of tensorial irreps in terms of sections of quantum super vector bundles over quantum projective superspaces.
\end{abstract}

\section{INTRODUCTION}

Quantized universal enveloping superalgebras [1] [2] ( which will be called quantum superalgebras for simplicity) represent the most important generalizations of the Drinfeld - Jimbo [3] quantized universal enveloping algebras. Their origin can be traced back to the Perk - Schultz solution of the Yang - Baxter equation and also the work of Bazhanov and Shadrikov [4]. However, systematical investigations of such algebraic structures only started about six years ago, but in an intensive manner. By now the subject has been developed quite extensively: the quasi - triangular Hopf superalgebraic structure of the quantum superalgebras was investigated [5]; the representation theory of large classes of quantum (affine)superalgebras and super Yangians was developed [6] [7]; applications of quantum superalgebras to integrable two dimensional models in statistical mechanics and quantum field theory were extensively explored [1] [8]. Quantum superalgebras have also been applied to the study of knot theory and 3 - manifolds [9] [10], yielding many new topological invariants, notably, the multi parameter generalizations of Alexander - Conway polynomials.

Closely related to the Drinfeld - Jimbo algebras are the quantum groups introduced by Woronowicz and Faddeev - Reshetikhin - Takhatajan [11, which are, in the spirit of Tannaka - Krein duality theory, the 'groups' associated with the quantized universal enveloping algebras. One very important aspect of quantum groups is their geometrical significance: they provide a concrete framework for developing noncommutative 
geometry [12, in particular, for investigating notions such as quantum flag varieties [13] and quantum fibre bundles.

Our aim here is to study the structure and representations of the quantum general linear supergroup $G L_{q}(m \mid n)$ in a systematical fashion by investigating the algebra of its representative functions. We start in section 2 with a concise treatment of finite dimensional unitary representations of $U_{q}(g l(m \mid n))$. Results will be repeatedly used in the remainder of the paper. In section 3 we define the the quantum general linear supergroup $G L_{q}(m \mid n)$, or more exactly, the superalgebra $G_{q}$ of functions on it. This is done by first defining the bi - superalgebras $G_{q}^{\pi}$ and $G_{q}^{\bar{\pi}}$, which are respectively generated by the matrix elements of the vector irrep and its dual irrep. Peter - Weyl type of bases for these bi - superalgebras are constructed. The $G_{q}$ is defined to be generated by $G_{q}^{\pi}$ and $G_{q}^{\bar{\pi}}$ with some extra relations. It has the structures of a * - Hopf superalgebra, which separates points of $U_{q}(g l(m \mid n))$, and factorizes into $G_{q}^{\pi} G_{q}^{\bar{\pi}}$. Section 4 treats the representation theory of the quantum supergroup, and in particular, parabolic induction. The geometrical interpretation of induced representations is discussed, leading naturally to the concepts of quantum homogeneous spaces and quantum super vector bundles. A quantum analog of Frobenius reciprocity is obtained; and a quantum version of the Borel - Weil theorem is proven for the covariant and contravariant tensorial irreps. Section 5 gives the explicit realizations of two infinite classes of tensorial irreps in terms of sections of quantum super vector bundles over the quantum projective superspace. In doing this, we also treat the quantum projective superspace in some detail.

\section{UNITARITY REPRESENTATIONS OF $U_{q}(g l(m \mid n))$}

The finite dimensional unitary representations of $U_{q}(g l(m \mid n))$ were classified in [15]. Here we will reformulate the results on the covariant and contravariant tensor irreps so that they can be readily used in the remainder of the paper. The material presented here also heavily relies on references [6] and [14].

\subsection{Hopf $*$ - superalgebras and unitary representations}

Let $A$ be a $\mathbb{Z}_{2}$ - graded associative algebra over the complex field $\mathbb{C}$. Its underlying $\mathbb{Z}_{2}$ - graded vector space is the direct sum $A=A_{0} \oplus A_{1}$ of the even subspace $A_{0}$ and the odd subspace $A_{1}$. We introduce the grading index [ ] : $A_{0} \cup A_{1} \rightarrow \mathbb{Z}_{2}$ such that $[a]=\theta$ if $a \in A_{\theta}$. We will call $A$ a $\mathbb{Z}_{2}$ - graded $*$ - algebra, or $*$ - superalgebra, if there exists an even anti - linear anti - automorphism $*: A \rightarrow A$ such that $* \circ *=i d_{A}$. We will denote $*(a)$ by $a^{*}$. Needless to say, $*(a b)=b^{*} a^{*}, a, b \in A$.

An important new feature of the $\mathbb{Z}_{2}$ - graded case is that for a given $*$ - operation of $A$, there exists an associated $*^{\prime}$ such that

$$
*^{\prime}(a)=(-1)^{[a]} a^{*},
$$

for $a$ being homogeneous, and extends to the whole of $A$ anti - linearly. There also exist the so called graded $*$ - operations, which, however, are not useful for this paper, thus will not be discussed any further.

Let $A$ and $B$ be two $\mathbb{Z}_{2}$ - graded * - algebras. Then $A \otimes_{\mathbb{C}} B$ has a natural $\mathbb{Z}_{2}$ graded $*$ - algebra structure, with the $*$ - operation defined for homogeneous elements 
by

$$
*(a \otimes b)=(-1)^{[a][b]} a^{*} \otimes b^{*}
$$

and for all the elements by extending this anti - linearly.

Consider a $\mathbb{Z}_{2}$ - graded Hopf algebra ( also called Hopf superalgebra ) H, with multiplication $m$, unit $1_{H}$, co - multiplication $\Delta$, co - unit $\epsilon$ and antipode $S$. We emphasize that the antipode is a linear anti- automorphism of the underlying algebra of $H$. In particular, for homogeneous $a, b \in A$, we have $S(a b)=(-1)^{[a][b]} S(b) S(a)$. $H$ will be called a $\mathbb{Z}_{2}$ - graded Hopf $*$ - algebra, or Hopf $*$ - superalgebra, if the underlying algebra of $H$ is a $*$ - superalgebra such that $\Delta$ and $\epsilon$ are $*$ - homomorphisms, i.e.,

$$
* \circ \Delta=\Delta \circ *, \quad * \circ \epsilon=\epsilon \circ *
$$

These properties together with the defining relations of the antipode

$$
m \circ(S \otimes i d) \Delta=m \circ(i d \otimes S) \Delta=1_{H} \epsilon
$$

imply that

$$
S \circ * \circ S \circ *=i d_{H}
$$

Let $V$ be a left $H$ - module. If there exists a non - degenerate sequilinear form $():, V \otimes V \rightarrow \mathbb{C}$, such that

$$
\begin{array}{cc}
(i) . \quad(a v, u)=\left(v, a^{*} u\right), & \forall u, v \in V, a \in H \\
(i i) . \quad & (v, v) \geq 0, \quad(v, v)=0 \text { iff } v=0
\end{array}
$$

we call $V$ and the associated representation of $H$ unitary.

Unitary representations have the following important properties

i). A unitary representation is completely reducible;

ii). The tensor product of two unitary ( with respect to the same * - operation) representations is again unitary;

iii). If a representation is unitary with respect to $*$, then its dual is unitary with respect to $*^{\prime}$.

All the three assertions are well known, but there are some related matters worth discussing. One is concerned with the requirement that two representations must be unitary with respect to the same $*$ - operation in order for their tensor product to be unitary as well. The tensor product $V \otimes_{\mathbb{C}} W$ of two $H$ - modules has a natural $H$ module structure

$$
a\{v \otimes w\}=\sum_{(a)}(-1)^{\left[a_{(2)}\right][v]} a_{(1)} v \otimes a_{(2)} w
$$

If both $V$ and $W$ are equipped with sequilinear forms $():, V \otimes_{\mathbb{C}} V \rightarrow \mathbb{C}$, and $():, W \otimes_{\mathbb{C}} W \rightarrow \mathbb{C}$, we can define a sequilinear form $(()):,\left(V \otimes_{\mathbb{C}} W\right)^{\otimes 2} \rightarrow \mathbb{C}$ by

$$
\left(\left(v_{1} \otimes w_{1}, v_{2} \otimes w_{2}\right)\right)=\left(v_{1}, v_{2}\right)\left(w_{1}, w_{2}\right)
$$


Now if both $V$ and $W$ are unitary with respect to the same $*$ - operation, then $(()$, is clearly positive definite and nondegenerate. Furthermore,

$$
\begin{aligned}
\left(\left(v_{1} \otimes w_{1}, a\left\{v_{2} \otimes w_{2}\right\}\right)\right) & =\sum_{(a)}(-1)^{\left[a_{(2)}\right]\left[v_{2}\right]}\left(\left(a_{(1)}^{*} v_{1} \otimes a_{(2)}^{*} w_{1}, v_{2} \otimes w_{2}\right)\right) \\
& =\left(\left(a^{*}\left\{v_{1} \otimes w_{1}\right\}, v_{2} \otimes w_{2}\right)\right) .
\end{aligned}
$$

Therefore, $V \otimes_{\mathbb{C}} W$ indeed furnishes a unitary $H$ - module. On the other hand, if, say, $V$ is $*$ - unitary, while $W$ is $*^{\prime}$ - unitary, then one can easily see that the above calculations will fail to go through.

The other concerns the third assertion, the validity of which actually requires some qualification, namely, the Hopf * - superalgebra $H$ in question must admit an even group like element $K_{2 \rho}$ satisfying

$$
K_{2 \rho}^{*}=K_{2 \rho}, \quad S^{2}(a)=K_{2 \rho} a K_{2 \rho}^{-1}, \quad \forall a \in H .
$$

Let $V$ be a locally finite module over $H$, which is unitary with respect to the sequilinear form $():, V \otimes V \rightarrow \mathbb{C}$. For every $v \in V$, we define $v^{\dagger}$ by $v^{\dagger}(w)=(v, w), \forall w \in V$, and denote the linear span of all such $v^{\dagger}$ by $V^{\dagger}$, which is a subspace of the dual vector space of $V$. The $V^{\dagger}$ has a natural $H$ module structure, with the action of $H$ given by

$$
\left(a v^{\dagger}\right)(w)=(-1)^{[a]\left[v^{\dagger}\right]} v^{\dagger}(S(a) w), \quad w \in V .
$$

Unitarity of $V$ leads to

$$
a v^{\dagger}=(-1)^{[a][v]}(* S(a) v)^{\dagger}
$$

We define a sequilinear form $(,)^{\prime}: V^{\dagger} \otimes V^{\dagger} \rightarrow \mathbb{C}$ by

$$
\left(v^{\dagger}, w^{\dagger}\right)^{\prime}=\left(K_{2 \rho} w, v\right)
$$

It follows from the properties of the original form on $V$ that $(,)^{\prime}$ is positive definite and nondegenerate. A straightforward calculation shows that

$$
\left(a v^{\dagger}, w^{\dagger}\right)^{\prime}=\left(v^{\dagger}, *^{\prime}(a) w^{\dagger}\right)^{\prime}
$$

where $*^{\prime}$ is defined by (四).

\section{$2.2 \quad U_{q}(g l(m \mid n))$}

Throughout the paper, we will denote by $\mathfrak{g}$ the complex Lie superalgebra $g l(m \mid n)$, and by $U(\mathfrak{g})$ its universal enveloping algebra. As is well known, there are the Drinfeld and Jimbo two versions of the quantized universal enveloping algebra $U_{q}(\mathfrak{g})$ of $\mathfrak{g}$, which, though, have very similar properties at generic $q$.

It is the Jimbo version of $U_{q}(\mathfrak{g})$ that will be used in this paper. Now $U_{q}(\mathfrak{g})$ is a $\mathbb{Z}_{2}$ graded unital associative algebra over $\mathbb{C}\left(q, q^{-1}\right), q$ being an indeterminate, generated by $\left\{K_{a}, K_{a}^{-1}, a \in \mathbf{I} ; E_{b b+1}, E_{b+1, b}, b \in \mathbf{I}^{\prime}\right\}, \mathbf{I}=\{1,2, \ldots, m+n\}, \mathbf{I}^{\prime}=\{1,2, \ldots, m+n-1\}$, 
subject to the following relations

$$
\begin{aligned}
& K_{a} K_{a}^{-1}=1, K_{a}^{ \pm 1} K_{b}^{ \pm 1}=K_{b}^{ \pm 1} K_{a}^{ \pm 1} \\
& K_{a} E_{b b \pm 1} K_{a}^{-1}= q_{a b b}^{\delta_{a b}-\delta_{a \pm 1} E_{b b \pm 1}} \\
& {\left[E_{a a+1}, E_{b+1 b}\right\}=} \delta_{a b}\left(K_{a} K_{a+1}^{-1}-K_{a}^{-1} K_{a+1}\right) /\left(q_{a}-q_{a}^{-1}\right), \\
&\left(E_{m m+1}\right)^{2}=\left(E_{m+1 m}\right)^{2}=0 \\
& E_{a a+1} E_{b b+1}= E_{b b+1} E_{a a+1}, \\
& E_{a+1 a} E_{b+1 b}= E_{b+1 b} E_{a+1 a}, \quad|a-b| \geq 2, \\
& \mathcal{S}_{a a \pm 1}^{(+)}= \mathcal{S}_{a a \pm 1}^{(-)}=0, \quad a \neq m, \\
&\left\{E_{m-1 m+2}, E_{m m+1}\right\}=\left\{E_{m+2 m-1}, E_{m+1 m}\right\}=0,
\end{aligned}
$$

where $q_{a}=q^{(-1)^{[a]}}$,

$$
\begin{aligned}
\mathcal{S}_{a a \pm 1}^{(+)} & =\left(E_{a a+1}\right)^{2} E_{a \pm 1 a+1 \pm 1}-\left(q+q^{-1}\right) E_{a a+1} E_{a \pm 1 a+1 \pm 1} E_{a a+1} \\
& +E_{a \pm 1 a+1 \pm 1}\left(E_{a a+1}\right)^{2} \\
\mathcal{S}_{a a \pm 1}^{(-)} & =\left(E_{a+1 a}\right)^{2} E_{a+1 \pm 1 a \pm 1}-\left(q+q^{-1}\right) E_{a+1 a} E_{a+1 \pm 1 a \pm 1} E_{a+1 a} \\
& +E_{a+1 \pm 1 a \pm 1}\left(E_{a+1 a}\right)^{2}
\end{aligned}
$$

and $E_{m-1 m+2}$ and $E_{m+2 m-1}$ are the $a=m-1, b=m+1$, cases of the following elements

$$
\begin{aligned}
E_{a b} & =E_{a c} E_{c b}-q_{c}^{-1} E_{c b} E_{a c}, \\
E_{b a} & =E_{b c} E_{c a}-q_{c} E_{c a} E_{b c}, \quad a<c<b .
\end{aligned}
$$

The $\mathbb{Z}_{2}$ grading of the algebra is specified such that the elements $K_{a}^{ \pm 1}, \forall a \in \mathbf{I}$, and $E_{b b+1}, E_{b+1 b}, b \neq m$, are even, while $E_{m m+1}$ and $E_{m+1 m}$ are odd. Above, we have also used the notation $[a]= \begin{cases}0, & \text { if } a \leq m, \\ 1, & \text { if } a>m .\end{cases}$

On the other hand, the Drinfeld version of $U_{q}(\mathfrak{g})$ is defined over $\mathbb{C}[[\hbar]], q=\exp (\hbar)$, and is completed with respect to the $\hbar$ - adic topology of $\mathbb{C}[[\hbar]]$. It is generated $\left\{E_{a a}, a \in \mathbf{I} ; E_{b b+1}, E_{b+1, b}, b \in \mathbf{I}^{\prime}\right\}$, subject to the same relations (3) with

$$
K_{a}=q_{a}^{E_{a a}}
$$

It is well known that $U_{q}(\mathfrak{g})$ has the structure of a $\mathbb{Z}_{2}$ graded Hopf algebra, with a co - multiplication

$$
\begin{aligned}
\Delta\left(E_{a a+1}\right) & =E_{a a+1} \otimes K_{a} K_{a+1}^{-1}+1 \otimes E_{a a+1}, \\
\Delta\left(E_{a+1 a}\right) & =E_{a+1 a} \otimes 1+K_{a}^{-1} K_{a+1} \otimes E_{a+1 a}, \\
\Delta\left(K_{a}^{ \pm 1}\right) & =K_{a}^{ \pm 1} \otimes K_{a}^{ \pm 1},
\end{aligned}
$$

co - unit

$$
\begin{aligned}
\epsilon\left(E_{a a+1}\right) & =E_{a+1 a}=0, \quad \forall a \in \mathbf{I}^{\prime} \\
\epsilon\left(K_{b}^{ \pm 1}\right) & =1, \quad \forall b \in \mathbf{I},
\end{aligned}
$$


and antipode

$$
\begin{aligned}
S\left(E_{a a+1}\right) & =-E_{a a+1} K_{a}^{-1} K_{a+1}, \\
S\left(E_{a+1 a}\right) & =-K_{a} K_{a+1}^{-1} E_{a+1 a}, \\
S\left(K_{a}^{ \pm 1}\right) & =K_{a}^{\mp 1} \otimes K_{a}^{\mp 1} .
\end{aligned}
$$

At generic $q$, the Jimbo version of $U_{q}(\mathfrak{g})$ has more or less the same representation theory as that of the Drinfeld version [6]. Let $\left\{\epsilon_{a} \mid a \in \mathbf{I}\right\}$ be the basis of a vector space with a bilinear for $\left(\epsilon_{a}, \epsilon_{b}\right)=(-1)^{[a]} \delta_{a b}$. The roots of the classical Lie superalgebra $g l(m \mid n)$ can be expressed as

$$
\epsilon_{a}-\epsilon_{b}, \quad a \neq b, \quad a, b \in \mathbf{I}
$$

For later use, we define

$$
2 \rho=\sum_{a \leq b}(-1)^{[a]+[b]}\left(\epsilon_{a}-\epsilon_{b}\right)
$$

From [6] we know that every finite dimensional irreducible $U_{q}(\mathfrak{g})$ module is of highest weight type and is essentially uniquely characterized by a highest weight. Let $W(\lambda)$ be an irreducible $U_{q}(\mathfrak{g})$ module with highest weight $\lambda=\sum_{a} \lambda_{a} \epsilon_{a}, \lambda_{a} \in \mathbb{C}$. There exists a unique ( up to scalar multiples) vector $v_{+}^{\lambda} \neq 0$ in $W(\lambda)$, called the highest weight vector, such that

$$
\begin{aligned}
E_{a a+1} v_{+}^{\lambda} & =0, \quad a \in \mathbf{I}^{\prime}, \\
K_{b} v_{+}^{\lambda} & =q_{b}^{\lambda_{b}} v_{+}^{\lambda}, \quad b \in \mathbf{I} .
\end{aligned}
$$

$W(\lambda)$ is finite dimensional if and only if $\lambda$ satisfies $\lambda_{a}-\lambda_{a+1} \in \mathbb{Z}_{+}, a \neq m$, and in that case, it has the same weight space decomposition as that of the corresponding irreducible $g l(m \mid n)$ module with the same highest weight.

\subsection{Unitarity of covariant and contravariant tensor irreps}

From this section on, we will assume that $U_{q}(\mathfrak{g})$ is obtained from the the Jimbo algebra by specializing $q$ to a real positive parameter different from 1 . To construct a $*-$ operation for $U_{q}(\mathfrak{g})$, we first consider the Hopf subalgebra generated by $e=E_{a a+1}$, $f=E_{a+1 a}$, and $k=K_{a} K_{a+1}^{-1}$, for a fixed $a \neq m$. It is not difficult to show that $*(e)=f k, *(f)=k^{-1} e, *\left(k^{ \pm 1}\right)=k^{ \pm 1}$ defines a $*$ - operation for this $U_{q}(s l(2))$ subalgebra. Possible generalizations of this to $U_{q}(\mathfrak{g})$ are

$$
\begin{aligned}
*\left(E_{a a+1}\right) & =(-1)^{(\theta+1) \delta_{m a}} E_{a+1 a} K_{a} K_{a+1}^{-1}, \\
*\left(E_{a+1 a}\right) & =(-1)^{(\theta+1) \delta_{m a}} K_{a}^{-1} K_{a+1} E_{a a+1}, \\
*\left(K_{a}^{ \pm 1}\right) & =K_{a}^{ \pm 1},
\end{aligned}
$$

where $\theta=1$ or 2 . It is quite obvious that the 'quadratic' relations of (3) are preserved by the $*$ - operations, and we have also explicitly checked that the 'Serre relations' are preserved as well. We will call the $*$ - operations type 1 and type 2 respectively when $\theta=1$ and 2 . 
It is also well known that

$$
K_{2 \rho}=\prod_{a<b}\left(K_{a} K_{b}^{-1}\right)^{(-1)^{[a]+[b]}}
$$

satisfies equation (2).

Now we consider the covariant and contravariant tensor irreps of $U_{q}(\mathfrak{g})$. The vector irrep $\pi$ of $U_{q}(\mathfrak{g})$ is of highest weight $\epsilon_{1}$. The corresponding module $\mathbb{E}$ has the standard basis $\left\{v_{a} \mid a \in \mathbf{I}\right\}$, such that

$$
\begin{aligned}
K_{a} v_{b} & =q_{a}^{\delta_{a b}} v_{b}, \\
E_{a a \pm 1} v_{b} & =\delta_{b a \pm 1} v_{a} .
\end{aligned}
$$

Define a sequilinear form on $\mathbb{E} \otimes \mathbb{E}$ by

$$
\left(v_{a}, v_{b}\right)=\delta_{a b} \prod_{c=1}^{a-1} q_{c}^{-1} .
$$

Then it is straightforward to show that with respect to the type $1 *$ - operation, we have

$$
\begin{aligned}
\left(E_{a a_{a \pm 1}} v_{b}, v_{c}\right) & =\left(v_{b}, E_{a a \pm 1}^{*} v_{c}\right) \\
\left(K_{a} v_{b}, v_{c}\right) & =\left(v_{b}, K_{a} v_{c}\right)
\end{aligned}
$$

Therefore, the vector irrep is unitary of type 1 .

The $U_{q}(\mathfrak{g})$ modules $\mathbb{E}^{\otimes k}, k \in \mathbb{Z}_{+}\left(\mathbb{E}^{0}=\mathbb{C}\right)$, obtained by repeated tensor products of the vector module with itself can be decomposed into direct sums of irreducible type 1 unitary modules, and we will call each direct summand an irreducible contravariant tensor module, and the corresponding irreducible representation a contravariant tensor irrep.

The contravariant tensor irreps can be characterized in the following way. Let $\mathbb{Z}_{+}$ be the set of nonnegative integers. Define a subset $\mathcal{P}$ of $\mathbb{Z}_{+}{ }^{\otimes(m+n)}$ by

$$
\mathcal{P}=\left\{p=\left(p_{1}, p_{2}, \ldots, p_{m+n}\right) \in \mathbb{Z}_{+}{ }^{\otimes(m+n)} \mid p_{m+1} \leq n, p_{a} \geq p_{a+1}, a \in \mathbf{I}^{\prime}\right\} .
$$

We associate with each $p \in \mathcal{P}$, a $\lambda^{(p)}=\sum_{a=1}^{m+n} \lambda_{a} \epsilon_{a}$ defined by

$$
\begin{aligned}
\lambda_{a}=p_{a}, & a \leq m, \\
\sum_{\mu=1}^{n} \lambda_{m+\mu} \epsilon_{m+\mu} & =\sum_{\nu=1}^{n} \sum_{\mu=1}^{p_{m+\nu}} \epsilon_{m+\mu} .
\end{aligned}
$$

Introduce the set

$$
\Lambda^{(1)}=\left\{\lambda^{(p)} \mid p \in \mathcal{P}\right\}
$$

From results of [15] [14] [6] we know that an irrep of $U_{q}(\mathfrak{g})$ is a contravariant tensor irrep if and only if its highest weight belongs to $\Lambda^{(1)}$. Needless to say, all such irreps are type 1 unitary.

Let $W(\lambda)$ be an irreducible contravariant tensor $U_{q}(\mathfrak{g})$ module with highest weight $\lambda \in \Lambda^{(1)}$. We define $\bar{\lambda}$ to be its lowest weight, and set $\lambda^{\dagger}=-\bar{\lambda}$. An explicit formula 
for $\lambda^{\dagger}$ was given in 14 ( section III. B. ), where a more compact characterization for the sets $\Lambda^{(1)}$ and $\Lambda^{(2)}$, which will be defined presently, was also given. We refer to that paper for details. Now the dual module $W(\lambda)^{\dagger}$ of $W(\lambda)$, which will we call a covariant tensor module, has highest weight $\lambda^{\dagger}$.

We introduce the set

$$
\Lambda^{(2)}=\left\{\lambda^{\dagger} \mid \lambda \in \Lambda^{(1)}\right\}
$$

An irrep is a covariant tensor irrep if and only if its highest weight is contained in $\Lambda^{(2)}$, and all covariant tensor irreps are unitary of type 2. The most important example of type 2 unitary modules is the covariant vector module $\mathbb{E}^{\dagger}$, which is the dual of the vector module $\mathbb{E}$. Its highest weight is given by $-\epsilon_{m+n}$.

We summarize our discussions into the following

Proposition 1 1. Each $U_{q}(\mathfrak{g})$ module $\mathbb{E}^{\otimes k}\left(\right.$ resp. $\left.\left(\mathbb{E}^{\dagger}\right)^{\otimes k}\right), k \in \mathbb{Z}_{+}$, can be decomposed into a direct sum of irreducible modules with highest weights belonging to $\Lambda^{(1)}\left(\operatorname{resp} . \Lambda^{(2)}\right)$.

2. Every irreducible $U_{q}(\mathfrak{g})$ module with highest weight belonging to $\Lambda^{(1)}$ (resp. $\left.\Lambda^{(2)}\right)$ is contained in some repeated tensor products of $\mathbb{E}\left(\right.$ resp. $\left.\mathbb{E}^{\dagger}\right)$ as an irreducible component.

More detailed structures of the covariant and contravariant tensor irreps can be understood, e.g., their characters and super characters can be computed, the Clebsch - Gordan problem of irreps within a given tensor type can also be resolved by using the supersymmetric Young diagram method. Here we elucidate some general aspects of the Clebsch - Gordan problem, which will play an important role in the remainder of the paper.

Denote by $[\lambda]$ the equivalence class of irreps with highest weight $\lambda$. For $\lambda$ and $\lambda^{\prime}$ both belonging to $\Lambda^{(1)}$, we interpret $[\lambda]+\left[\lambda^{\prime}\right]$ as the equivalence class of the direct sum representations, and $[\lambda] \cdot\left[\lambda^{\prime}\right]$ as that of the direct products. Let $\left[\Lambda^{(1)}\right]$ be the $\mathbb{Z}^{+}$ module with a basis $\left\{[\lambda] \mid \lambda \in \Lambda^{(1)}\right\}$. Then the '.' operation defines a multiplication on $\left[\Lambda^{(1)}\right]$. Clearly $[\lambda] \cdot[0]=[0] \cdot[\lambda]=[\lambda]$. Further more, from section $\mathrm{V}$ of [14 we can deduce that if $[\lambda] \cdot\left[\lambda^{\prime}\right]=\left[\lambda^{1}\right]+\left[\lambda^{2}\right]+\ldots+\left[\lambda^{k}\right]$, then none of the $\lambda^{i}$ is zero unless both $\lambda$ and $\lambda^{\prime}$ are zero. This is in agreement with the fact that

$$
\Lambda^{(1)} \bigcap \Lambda^{(2)}=\{0\}
$$

The dicussions above can be repeated word by word for the irreps with highest weights belonging to $\Lambda^{(2)}$.

\section{QUANTUM SPECIAL LINEAR SUPERGROUP $G L_{q}(m \mid n)$}

For compact Lie groups in the classical setting, there exists the celebrated Tannaka - Krein duality theory [17], which enables the reconstruction of a group from the Hopf algebra of its representative functions. The theory of quantum groups [11] makes essential use of a quantum analog of the duality [16], and is formulated entirely in terms of the algebra of functions. We will adopt the same philosophy here to formulate and 
study quantum supergroups. However, we should mention that Lie supergroups are much more complicated than ordinary compact Lie groups in structures; at the best, the Tannaka - Krein duality holds in a restricted sense for Lie supergroups even at the classical situation, though we have not come across any treatment of the problem in the literature.

\subsection{Subalgebra of functions associated with the vector irrep}

As before, we denote by $\pi$ the vector irrep of $U_{q}(\mathfrak{g})$ relative to the standard basis $\left\{v_{a} \mid a \in \mathbf{I}\right\}$ of $\mathbb{E}$. Then

$$
x v_{a}=\sum_{b} \pi(x)_{b a} v_{b}, \quad x \in U_{q}(\mathfrak{g}) .
$$

Let $\left(U_{q}(\mathfrak{g})\right)^{0}$ be the finite dual of $U_{q}(\mathfrak{g})$. Consider the elements $t_{a b}, a, b \in \mathbf{I}$ of $\left(U_{q}(\mathfrak{g})\right)^{0}$ satisfying

$$
t_{a b}(x)=\pi(x)_{a b}, \quad \forall x \in U_{q}(\mathfrak{g}) .
$$

It is easy to show that the $t_{a b}$ indeed belong to $\left(U_{q}(\mathfrak{g})\right)^{0}$. Also note that $t_{a b}$ is even if $[a]+[b] \equiv 0(\bmod 2)$, and odd otherwise.

Standard Hopf algebra theory asserts that $\left(U_{q}(\mathfrak{g})\right)^{0}$ is a $\mathbb{Z}_{2}$ - graded Hopf algebra with its structures dualizing those of $U_{q}(\mathfrak{g})$. Consider the subalgebra $G_{q}^{\pi}$ of $\left(U_{q}(\mathfrak{g})\right)^{0}$ generated by $t_{a b}, a, b \in \mathbf{I}$. The multiplication which $G_{q}^{\pi}$ inherits from $\left(U_{q}(\mathfrak{g})\right)^{0}$ is given by

$$
\begin{aligned}
\left\langle t t^{\prime}, x\right\rangle & =\sum_{(x)}\left\langle t \otimes t^{\prime}, x_{(1)} \otimes x_{(2)}\right\rangle \\
& =\sum_{(x)}(-1)^{\left[t^{\prime}\right]\left[x_{(1)}\right]}\left\langle t, x_{(1)}\right\rangle\left\langle t, x_{(2)}\right\rangle, \quad \forall t, t^{\prime} \in G_{q}^{\pi}, x \in U_{q}(\mathfrak{g}) .
\end{aligned}
$$

To better understand the algebraic structure of $G_{q}^{\pi}$, we recall that the Drinfeld version of $U_{q}(\mathfrak{g})$ admits a universal $R$ matrix, which in particular satisfies

$$
R \Delta(x)=\Delta^{\prime}(x) R, \quad \forall x \in U_{q}(\mathfrak{g}) .
$$

Applying $\pi \otimes \pi$ to both sides of the equation yields

$$
R^{\pi \pi}(\pi \otimes \pi) \Delta(x)=(\pi \otimes \pi) \Delta^{\prime}(x) R^{\pi \pi},
$$

where

$$
\begin{aligned}
R^{\pi \pi} & :=(\pi \otimes \pi) R \\
& =q^{\sum_{a \in \mathbf{I}} e_{a a} \otimes e_{a a}(-1)^{[a]}}+\left(q-q^{-1}\right) \sum_{a<b} e_{a b} \otimes e_{b a}(-1)^{[b]}
\end{aligned}
$$

It is important to realize that equation (8) makes perfect sense within the Jimbo formulation of the quantized universal enveloping algebra $U_{q}(\mathfrak{g})$, even when $q$ is specialized to a real parameter. We can re - interpret the equation in terms of the $t_{a b}$. Set $t=\sum_{a, b} e_{a b} \otimes t_{a b}$. Then

$$
R_{12}^{\pi \pi} t_{1} t_{2}=t_{2} t_{1} R_{12}^{\pi \pi} .
$$


The co - multiplication $\Delta$ of $G_{q}^{\pi}$ is also defined in the standard way by

$$
\left\langle\Delta\left(t_{a b}\right), x \otimes y\right\rangle=\left\langle t_{a b}, x y\right\rangle=\pi(x y)_{a b}, \quad \forall x, y \in U_{q}(\mathfrak{g}) .
$$

We have

$$
\Delta\left(t_{a b}\right)=\sum_{c \in \mathbf{I}}(-1)^{([a]+[c])([c]+[b])} t_{a c} \otimes t_{c b} .
$$

$G_{q}^{\pi}$ also has the unit $\epsilon$, and the co - unit $1_{U_{q}(\mathfrak{g})}$. Therefore, $G_{q}^{\pi}$ has the structures of a $\mathbb{Z}_{2}$ - graded bi - algebra. However, it does not admit an antipode, as will be explained later.

Let $\pi^{(\lambda)}$ be an arbitrary irreducible contravariant tensor representation of $U_{q}(\mathfrak{g})$. We may also regard $\pi^{(\lambda)}$ as a representative of $[\lambda]$, where $\lambda \in \Lambda^{(1)}$. Define the elements $t_{i j}^{(\lambda)}, i, j=1,2, \ldots, \operatorname{dim}_{\mathbb{C}} \pi^{(\lambda)}$, of $\left(U_{q}(\mathfrak{g})\right)^{0}$ by

$$
t_{i j}^{(\lambda)}(x)=\pi^{(\lambda)}(x)_{i j}, \quad \forall x \in U_{q}(\mathfrak{g}) .
$$

It is an immediate consequence of Proposition [ that $t_{i j}^{(\lambda)} \in G_{q}^{\pi}$, for all $i, j$ and $\lambda \in \Lambda^{(1)}$, and every $f \in G_{q}^{\pi}$ can be expressed as a linear sum of these elements. From the representation theory of $U_{q}(\mathfrak{g})$ we can deduce that these elements are also linearly independent. Introduce the vector spaces

$$
T^{(\lambda)}=\bigoplus_{i, j=1}^{\operatorname{dim} \pi^{(\lambda)}} \mathbb{C} t_{i j}^{(\lambda)}
$$

Then

Proposition 2 As a vector space,

$$
G_{q}^{\pi}=\bigoplus_{\lambda \in \Lambda^{(1)}} T^{(\lambda)}
$$

Let us also denote the antipode of $\left(U_{q}(\mathfrak{g})\right)^{0}$ by $S$. Then for any $t_{i j}^{(\lambda)} \in G_{q}^{\pi}$,

$$
S\left(t_{i j}^{(\lambda)}\right)(x)=t_{i j}^{(\lambda)}(S(x)), \quad \forall x \in U_{q}(\mathfrak{g}) .
$$

That is, $S\left(t_{i j}^{(\lambda)}\right)$ are the matrix elements of the dual irrep of $\pi^{(\lambda)}$, the highest weight of which is not contained in $\Lambda^{(1)}$ unless $\lambda=0$. Therefore, $G_{q}^{\pi}$ by itself does not admit an antipode.

3.2 Subalgebra of functions associated with the dual vector irrep

Let $\left\{\bar{v}_{a} \mid a \in \mathbf{I}\right\}$ be the basis of $\mathbb{E}^{\dagger}$ dual to the standard basis of $\mathbb{E}$, i.e.,

$$
\bar{v}_{a}\left(v_{b}\right)=\delta_{a b} .
$$

Denote by $\bar{\pi}$ the covariant vector irrep relative to this basis. Let $\bar{t}_{a b}, a, b \in \mathbf{I}$, be the elements of $\left(U_{q}(\mathfrak{g})\right)^{0}$ such that

$$
\bar{t}_{a b}(x)=\bar{\pi}(x)_{a b}, \quad \forall x \in U_{q}(\mathfrak{g}) .
$$


Note that $\bar{t}_{a b}$ is even if $[a]+[b] \equiv 0(\bmod 2)$, and odd otherwise. These elements generate a $\mathbb{Z}_{2}$ - graded bi - subalgebra $G_{q}^{\bar{\pi}}$ of $\left(U_{q}(\mathfrak{g})\right)^{0}$ in the standard fashion. Here we merely point out that they obey the relation

$$
R_{12}^{\bar{\pi} \bar{\pi}} \bar{t}_{1} \bar{t}_{2}=\bar{t}_{2} \bar{t}_{1} R_{12}^{\bar{\pi} \bar{\pi}}
$$

where

$$
\begin{aligned}
\bar{t} & =\sum_{a, b} e_{a b} \otimes \bar{t}_{b a}, \\
R^{\bar{\pi} \bar{\pi}} & =(\bar{\pi} \otimes \bar{\pi}) R, \\
& =q^{\sum_{a \in \mathbf{I}} e_{a a} \otimes e_{a a}(-1)^{[a]}}+\left(q-q^{-1}\right) \sum_{a>b} e_{a b} \otimes e_{b a}(-1)^{[b]} .
\end{aligned}
$$

Also, the co - multiplication is given by

$$
\Delta\left(\bar{t}_{a b}\right)=\sum_{c \in \mathbf{I}}(-1)^{([a]+[c])([c]+[b])} \bar{t}_{a c} \otimes \bar{t}_{c b} .
$$

Denote by $\bar{\pi}^{(-\bar{\lambda})}$ the irrep dual to $\pi^{(\lambda)}, \lambda \in \Lambda^{(1)}$, in a given homogeneous basis. Introduce the elements $\bar{t}_{i j}^{(-\bar{\lambda})}, i, j=1,2, \ldots, \operatorname{dim}_{\mathbb{C}} \pi^{(\lambda)}$, of $\left(U_{q}(\mathfrak{g})\right)^{0}$ such that

$$
\bar{t}_{i j}^{(-\bar{\lambda})}(x)=\bar{\pi}_{i j}^{(-\bar{\lambda})}(x), \quad \forall x \in U_{q}(\mathfrak{g}) .
$$

Then it follows from Proposition 1 that these elements form a basis of $G_{q}^{\bar{\pi}}$. Set $\bar{T}^{(\mu)}$ $=\oplus_{i, j} \mathbb{C}_{i j}^{(\mu)}$, we have

\section{Proposition 3}

$$
G_{q}^{\bar{\pi}}=\bigoplus_{\mu \in \Lambda^{(2)}} \bar{T}^{(\mu)}
$$

\subsection{Algebra $G_{q}$ of functions on $G L_{q}(m \mid n)$}

We define the algebra $G_{q}$ of functions on the quantum general linear supergroup $G L_{q}(m \mid n)$ to be the $\mathbb{Z}_{2}$ - graded subalgebra of $\left(U_{q}(\mathfrak{g})\right)^{0}$ generated by $\left\{t_{a b}, \bar{t}_{a b} \mid a, b \in \mathbf{I}\right\}$. The $t_{a b}$ and $\bar{t}_{a b}$, besides obeying the the relations (9) and (11), also satisfy

$$
R_{12}^{\bar{\pi} \pi} \bar{t}_{1} t_{2}=t_{2} \bar{t}_{1} R_{12}^{\bar{\pi} \pi}
$$

where

$$
\begin{aligned}
R^{\bar{\pi} \pi} & :=(\bar{\pi} \otimes \pi) R \\
& =q^{-\sum_{a \in \mathbf{I}} e_{a a} \otimes e_{a} a(-1)^{[a]}}-\left(q-q^{-1}\right) \sum_{a<b} e_{b a} \otimes e_{b a}(-1)^{[a]+[b]+[a][b]} .
\end{aligned}
$$

Equation (12) enables us to factorize $G_{q}$ into

$$
G_{q}=G_{q}^{\pi} G_{q}^{\bar{\pi}}
$$

As both $G_{q}^{\pi}$ and $G_{q}^{\bar{\pi}}$ are $\mathbb{Z}_{2}$ - graded bi - algebras, $G_{q}$ inherits a natural bi - algebra structure. It also admits an antipode. By considering

$$
\left(x \bar{v}_{a}\right)\left(v_{b}\right)=(-1)^{[x][a]} \bar{v}_{a}\left(S(x) v_{b}\right), \quad x \in U_{q}(\mathfrak{g}),
$$

where $\left\{v_{a}\right\}$ is the standard basis of the vector irrep, and $\left\{\bar{v}_{a}\right\}$ is the basis of the covariant vector irrep dual to $\left\{v_{a}\right\}$, we arrive at 
Lemma 1 The antipode $S: G_{q} \rightarrow G_{q}$ is a linear anti-automorphism given by

$$
\begin{aligned}
& S\left(t_{a b}\right)=(-1)^{[a][b]+[a]} \bar{t}_{b a}, \\
& S\left(\bar{t}_{a b}\right)=(-1)^{[a][b]+[b]} q^{\left(2 \rho, \epsilon_{a}-\epsilon_{b}\right)} t_{b a} .
\end{aligned}
$$

Therefore, $G_{q}$ has the structures of a $\mathbb{Z}_{2}$ - graded Hopf algebra.

Furthermore, * - operations can also be constructed for $G_{q}$, thus turning it into a Hopf $*$ - superalgebra. We have

$$
\begin{aligned}
& *\left(t_{a b}\right)=(-1)^{(\theta+[a])([a]+[b])} \bar{t}_{a b} \\
& *\left(\bar{t}_{a b}\right)=(-1)^{(\theta+[a])([a]+[b])} t_{a b} .
\end{aligned}
$$

where $\theta \in \mathbb{Z}_{2}$.

An important property of $G_{q}$ is that it separates points of $U_{q}(\mathfrak{g})$, that is, for any nonvanishing $x \in U_{q}(\mathfrak{g})$, there exists $f \in G_{q}$ such that $f(x) \neq 0$. As a matter of fact, $G_{q}^{\pi}$ by itself separates points of $U_{q}(\mathfrak{g})$. Put differently, for any $u \in U_{q}(\mathfrak{g})$, if $u \neq 0$, then $\pi^{\otimes p}(u) \neq 0$ for some $p \in \mathbb{Z}_{+}$.

To verify our assertion, we first consider the corresponding proposition in the classical situation of $U(\mathfrak{g})$ in detail. Let $E_{a b}^{(0)}, a, b \in \mathbf{I}$, be the standard generators of $\mathfrak{g}$ embedded in its universal enveloping algebra. In the vector irrep $\pi^{(0)}$, one has

$$
\pi^{(0)}\left(E_{a b}^{(0)}\right)=e_{a b}
$$

We isolate the $u(1)$ subalgebra of $\mathfrak{g}$ with the generator

$$
Z^{(0)}=\sum_{a \in \mathbf{I}} E_{a a}^{(0)}
$$

and denote by $X_{A}^{(0)}, A=1, \ldots,(m+n)^{2}-1$, the elements $E_{c c}^{(0)}-E_{c+1 c+1}^{(0)}, c \in \mathbf{I}^{\prime}$, and $E_{a b}^{(0)}, a \neq b$, in any fixed ordering. Then a PBW basis for $U(\mathfrak{g})$ is given by,

$$
\left\{B_{k, A_{1} \ldots A_{l}}^{(0)}=\left(Z^{(0)}\right)^{k} X_{A_{1}}^{(0)} \ldots X_{A_{l}}^{(0)} \mid k, l \in \mathbb{Z}_{+}, A_{i} \leq A_{i+1}, A_{i} \neq A_{i+1} \text { if }\left[X_{A_{i}}^{(0)}\right]=1\right\} .
$$

Set $\pi^{(0)}\left(X_{A}^{(0)}\right)=e_{A}$. Denote by $\mathcal{M}$ the vector space of $(m+n) \times(m+n)$ matrices, and define

$$
\mathcal{R}_{k}=\sum_{i=0}^{k-1} \underbrace{\mathcal{M} \otimes \ldots \otimes \mathcal{M}}_{i} \otimes I \otimes \underbrace{\mathcal{M} \otimes \ldots \otimes \mathcal{M}}_{k-1-i} .
$$

Let

$$
b_{A_{1} \ldots A_{k}}=\sum_{\sigma \in S_{k}}(-1)^{\left|\sigma_{\{A\}}\right|} e_{A_{\sigma(1)}} \otimes e_{A_{\sigma(2)}} \otimes \ldots \otimes e_{A_{\sigma(k)}}
$$

where $\left|\sigma_{\{A\}}\right|$ is the number of permutations required amongst odd elements in order to change $X_{A_{1}} \otimes X_{A_{2}} \otimes \ldots \otimes X_{A_{k}}$ to $X_{A_{\sigma(1)}} \otimes X_{A_{\sigma(1)}} \otimes \ldots \otimes X_{A_{\sigma(k)}}$. Clearly, the elements

$$
\left\{b_{A_{1} \ldots A_{k}} \mid k \in \mathbb{Z}_{+}, A_{i} \leq A_{i+1}, A_{i} \neq A_{i+1} \text { if }\left[X_{A_{i}}\right]=1\right\}
$$


are linearly independent in $\mathcal{M}^{\otimes k}$, and we will denote by $\mathcal{L}_{k}$ their linear span. By considering the trace ( not the supertrace! ) on each factor of $\mathcal{M}^{\otimes k}$, we can easily see that $\mathcal{L}_{k}$ intersects $\mathcal{R}_{k}$ trivially. Therefore,

$$
\begin{aligned}
\left(\pi^{(0)}\right)^{\otimes(k+p)}\left(B_{0, A_{1} \ldots A_{k}}^{(0)}\right) & =\left(\left(\pi^{(0)}\right)^{\otimes k} \otimes\left(\pi^{(0)}\right)^{\otimes p}\right)\left(B_{0, A_{1} \ldots A_{k}}^{(0)}\right) \\
& =b_{A_{1} \ldots A_{k}} \otimes I^{\otimes p}+r_{k, p}, \quad r_{k, p} \in \mathcal{R}_{k} \otimes \mathcal{M}^{\otimes p}
\end{aligned}
$$

are linearly indepenedent as elements of $\mathcal{M}^{\otimes(k+p)}$.

Consider $u \in U(\mathfrak{g})$ given by

$$
u=\sum_{k=0}^{K} \sum_{l=0}^{L} \sum_{\{A\}} C_{k}, A_{1} \ldots A_{l} B_{k, A_{1} \ldots A_{l}}^{(0)}, \quad C_{k}, A_{1} \ldots A_{l} \in \mathbb{C} .
$$

Using

$$
\left(\pi^{(0)}\right)^{\otimes p}\left(Z^{k}\right)=p^{k} I^{\otimes p}
$$

we immediately see that $\left(\pi^{(0)}\right)^{\otimes p}(u)=0, \forall p>L$, requires

$$
\sum_{k=0}^{K} p^{k} C_{k}, A_{1} \ldots A_{l}=0, \quad \forall p>L
$$

which forces all the $C_{k}, A_{1} \ldots A_{l}$ to vanish. This completes the proof for the classical case.

Remark: There is something slightly unnatural about our proof, that is, the combination $E_{m m}^{(0)}-E_{m+1 m+1}^{(0)}$ does not belong to $\operatorname{sl}(m \mid n) \subset \mathfrak{g}$, and this in turn forced us to consider the ordinary trace instead of the supertrace in proving $\mathcal{L}_{k} \cap \mathcal{R}_{k}=\{0\}$. We can avoid this unnaturalness when $m \neq n$ by using $E_{m m}^{(0)}+E_{m+1 m+1}^{(0)}$ instead, but not when $m=n$.

With the above preparations we can now readily prove our assertion for the quantum superalgebra. We first consider the Drinfeld version of $U_{q}(\mathfrak{g})$. Similar to the classical case, we set

$$
Z=\sum_{a \in \mathbf{I}} E_{a a}
$$

and denote by $X_{A}, A=1, \ldots,(m+n)^{2}-1$, the elements $E_{c c}-E_{c+1} c+1, c \in \mathbf{I}^{\prime}$ and $E_{a b}, a \neq b$, in a fixed ordering. Then

$$
\left\{B_{k, A_{1} \ldots A_{l}}=Z^{k} X_{A_{1}} \ldots X_{A_{l}} \mid k, l \in \mathbb{Z}_{+}, A_{i} \leq A_{i+1}, A_{i} \neq A_{i+1} \text { if }\left[X_{A_{i}}\right]=1\right\}
$$

forms a PBW basis for $U_{q}(\mathfrak{g})$. Given

$$
u=\hbar^{k}\left(u_{0}+\hbar u_{1}+\hbar^{2} u_{2}+\ldots\right)
$$

Each $u_{i}$ is a finite $\mathbb{C}$ - combination of some $B_{k}, A_{1} \ldots A_{l}$, and $u_{0}$ is assumed to be nonzero. Then it follows from the classical case that there exist infinitely many $p \in \mathbb{Z}_{+}$such that

$$
\pi^{\otimes p}(u) \not \equiv 0\left(\bmod \hbar^{k+1}\right)
$$


For the Jimbo algebra, we observe that ordered monomials in $E_{a b}, a \neq b$, and $K_{a}^{ \pm 1}$ form a basis of $U_{q}(\mathfrak{g})$. Given $u \in U_{q}(\mathfrak{g})$, and a positive integer $p$, we consider the matrix elements of $\left.\pi^{\otimes p}(u)\right|_{q=\exp (\hbar)}$ as power series in $\hbar . \pi^{\otimes p}(u) \neq 0$ if and only if some of these power series do not vanish identically. Now for the purpose of computing $\left.\pi^{\otimes p}(u)\right|_{q=\exp (\hbar)}$, we can make the identification

$$
\begin{aligned}
\pi^{\otimes p}\left(K_{a}\right) & =\sum_{k=0}^{\infty} \frac{(-1)^{k[a]} \hbar^{k}}{k !} e_{a a}(p)^{k}, \\
e_{a a}(p) & =\sum_{i=0}^{p-1} \underbrace{I \otimes \ldots \otimes I}_{i} \otimes e_{a a} \otimes \underbrace{I \otimes \ldots \otimes I}_{p-i-1} .
\end{aligned}
$$

This takes us back to the Drinfeld algebra situation, and we have already shown that in that situation the $\pi^{\otimes p}, p \in \mathbb{Z}_{+}$, separates points of $U_{q}(\mathfrak{g})$.

We summarize the discussions of this section into a Proposition, points ii) and iii) of which may be considered as a partial generalization of the classical Peter - Weyl theorem to the quantum supergroup in an algebraic setting:

Proposition 4 i) $G_{q}$ is a* - Hopf superalgebra;

ii). $G_{q}$ separates points of $U_{q}(\mathfrak{g})$;

iii). The following elements span $G_{q}$ :

$$
\begin{array}{ll}
t_{i j}^{(\lambda)} \bar{t}_{i^{\prime} j^{\prime}}^{(\mu)}, \quad & i, j=1,2, \ldots, \operatorname{dim} \pi^{(\lambda)}, \quad \lambda \in \Lambda^{(1)}, \\
& i^{\prime}, j^{\prime}=1,2, \ldots, \operatorname{dim} \bar{\pi}^{(\mu)}, \quad \mu \in \Lambda^{(2)} .
\end{array}
$$

However, we should point out that these elements are not linearly independent.

\section{INDUCED REPRESENTATIONS OF $G_{q}$}

We will develop parabolic induction for representations of $G_{q}$ in this section. Recall that corresponding to every locally finite right co - module $\omega: W \rightarrow W \otimes G_{q}$ over $G_{q}$, there exists a unique left $U_{q}(\mathfrak{g})$ module $U_{q}(\mathfrak{g}) \otimes W \rightarrow W$ with the module action defined by

$$
x w=\omega(w)(x), \quad x \in U_{q}(\mathfrak{g}), \quad w \in W .
$$

A similar correspondence exists for left $G_{q}$ co - modules and right $U_{q}$ modules. Therefore, we can describe the representation theory of $G_{q}$ in both the $G_{q}$ co - module language and $U_{q}(\mathfrak{g})$ module language, depending on which one is more convenient in a given situation. We will largely use the latter here.

4.1 Parabolic subalgebras of $U_{q}(g l(m \mid n))$

Let $\Theta$ be a subset of $\mathbf{I}^{\prime}$. Introduce the following sets of elements of $U_{q}(\mathfrak{g})$ :

$$
\begin{aligned}
\mathcal{S}_{l} & =\left\{K_{a}^{ \pm 1}, a \in \mathbf{I} ; \quad E_{c c+1}, E_{c+1}, c \in \boldsymbol{\Theta}\right\} ; \\
\mathcal{S}_{p_{+}} & =\mathcal{S}_{l} \cup\left\{E_{c c+1}, c \in \mathbf{I}^{\prime} \backslash \boldsymbol{\Theta}\right\} ; \\
\mathcal{S}_{p_{-}} & =\mathcal{S}_{l} \cup\left\{E_{c+1} c, c \in \mathbf{I}^{\prime} \backslash \boldsymbol{\Theta}\right\} .
\end{aligned}
$$


The elements of each set generate a $\mathbb{Z}_{2}$ - graded Hopf subalgebra of $U_{q}(\mathfrak{g})$. We denote by $U_{q}(\mathfrak{l})$ the Hopf subalgebra generated by the elements of $\mathcal{S}_{l}$, and by $U_{q}\left(\mathfrak{p}_{ \pm}\right)$the Hopf subalgebras respectively generated by the elements of $\mathcal{S}_{p_{ \pm}}$. In the classical limit, the Hopf subalgebras $U_{q}\left(\mathfrak{p}_{ \pm}\right)$coincide with the universal enveloping algebras of parabolic subalgebras of the Lie superalgebra $\mathfrak{g}$. Therefore, we will call $U_{q}\left(\mathfrak{p}_{ \pm}\right)$parabolic subalgebras of $U_{q}(\mathfrak{g})$.

Let $V_{\mu}$ be a finite dimensional irreducible $U_{q}(\mathfrak{l})$ module. Then $V_{\mu}$ is of highest weight type. Let $\mu$ be the highest weight and $\tilde{\mu}$ the lowest weight of $V_{\mu}$ respectively. We can extend $V_{\mu}$ in a unique fashion to a $U_{q}\left(\mathfrak{p}_{+}\right)$module, which we still denote by $V_{\mu}$, such that the elements of $\mathcal{S}_{p_{+}} \backslash \mathcal{S}_{l}$ act by zero. Similarly, $V_{\mu}$ also leads to a $U_{q}\left(\mathfrak{p}_{-}\right)$module, on which the elements of $\mathcal{S}_{p_{-}} \backslash \mathcal{S}_{l}$ act by zero. It is not difficult to see that all finite dimensional irreducible $U_{q}\left(\mathfrak{p}_{ \pm}\right)$modules are of this kind.

Consider a finite dimensional irreducible $U_{q}(\mathfrak{g})$ module $W(\lambda)$ with highest weight $\lambda$ and lowest weight $\bar{\lambda}$. $W(\lambda)$ can be restricted into a $U_{q}\left(\mathfrak{p}_{+}\right)$or $U_{q}\left(\mathfrak{p}_{-}\right)$module in a natural way, and the resultant module is always indecomposable, but not irreducible in general.

Consider first the case of $U_{q}\left(\mathfrak{p}_{+}\right)$. We wish to examine the $\mathbb{Z}_{2}$ - graded vector space $\operatorname{Hom}_{U_{q}\left(\mathfrak{p}_{+}\right)}\left(W(\lambda), V_{\mu}\right)$, which graded - commutes with $U_{q}\left(\mathfrak{p}_{+}\right)$, namely,

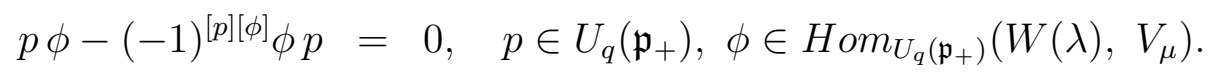

Because of the irreducibility of $V_{\mu}$, every non - zero $\phi \in \operatorname{Hom}_{U_{q}\left(\mathfrak{p}_{+}\right)}\left(W(\lambda), V_{\mu}\right)$ must be surjective, and thus $V_{\mu} \cong W(\lambda) / \operatorname{Ker} \phi$. As a $U_{q}\left(\mathfrak{p}_{+}\right)$module, $W(\lambda)$ is indecomposable, and contains a unique maximal proper submodule $M$ such that the lowest weight vector $w_{-}$of $W(\lambda)$ does not belong to $M$. Therefore, $\operatorname{Ker} \phi=M$, and $V_{\mu}=\phi\left(U_{q}(\mathfrak{l}) w_{-}\right)$. This forces $\bar{\lambda}=\tilde{\mu}$, and all elements of $\operatorname{Hom}_{U_{q}\left(\mathfrak{p}_{+}\right)}\left(W(\lambda), V_{\mu}\right)$ are scalar multiples of one another. It is worth observing that the map $\phi$ may be odd. In fact its degree is given by $[\phi] \equiv\left[w_{-}\right]+\left[\phi\left(w_{-}\right)\right](\bmod 2)$. The case of $U_{q}\left(\mathfrak{p}_{-}\right)$can be studied in exactly the same way. To summarize, we have

\section{Lemma 2}

$$
\begin{aligned}
\operatorname{dim}_{\mathbb{C}} \operatorname{Hom}_{U_{q}\left(\mathfrak{p}_{+}\right)}\left(W(\lambda), V_{\mu}\right) & = \begin{cases}1, & \bar{\lambda}=\tilde{\mu}, \\
0, & \bar{\lambda} \neq \tilde{\mu}\end{cases} \\
\operatorname{dim}_{\mathbb{C}} \operatorname{Hom}_{U_{q}\left(\mathfrak{p}_{-}\right)}\left(W(\lambda), V_{\mu}\right) & = \begin{cases}1, & \lambda=\mu, \\
0, & \lambda \neq \mu .\end{cases}
\end{aligned}
$$

\subsection{Induced representations and quantum superbundles}

Let us first introduce two types of left actions of $U_{q}(\mathfrak{g})$ on $G_{q}$, which correspond to the left and right translations in the classical situation.

Define a bilinear map $\cdot: U_{q}(\mathfrak{g}) \otimes G_{q} \rightarrow G_{q}$ by

$$
\begin{aligned}
x \otimes f & \mapsto x \cdot f \\
& =\sum_{(f)}\left\langle f_{(1)}, S^{-1}(x)\right\rangle f_{(2)},
\end{aligned}
$$

which can be easily shown to satisfy

$$
\begin{aligned}
& (x \cdot f)(y)=(-1)^{[x][y]} f\left(S^{-1}(x) y\right), \\
& x \cdot(y \cdot f)=(x y) \cdot f, \quad x, y \in U_{q}(\mathfrak{g}), f \in G_{q} .
\end{aligned}
$$


(We assume that the elements $x, y \in U_{q}(\mathfrak{g})$ and $g, f \in G_{q}$ are homogeneous for the sake of simplicity. All the statements below generalize to inhomogeneous elements in the obvious way.) Therefore, this defines a left action of $U_{q}(\mathfrak{g})$ on $G_{q}$, which corresponds to the left translation of Lie groups in the classical situation. It is worth observing that we may replace $S^{-1}$ in the above definition, and arrive at a different left action.

Another left action 'o' of $U_{q}(\mathfrak{g})$ on $G_{q}$ can be defined by

$$
x \circ f=\sum_{(f)} f_{(1)}(-1)^{[x]([f]+[x])}\left\langle f_{(2)}, x\right\rangle .
$$

Straightforward calculations can show that

$$
\begin{aligned}
y \circ(x \circ f) & =(x y) \circ f ; \\
(x \circ f)(y) & =f(y x), \\
\left(i d_{G_{q}} \otimes x \circ\right) \Delta(f) & =\Delta(x \circ f) .
\end{aligned}
$$

This corresponds to the right translation in the classical theory. It graded - commutes with the action ' ', namely,

$$
x \circ(y \cdot f)=(-1)^{[x][y]} y \cdot(x \circ f) .
$$

Let $U_{q}(\mathfrak{p})$ denote either $U_{q}\left(\mathfrak{p}_{+}\right)$or $U_{q}\left(\mathfrak{p}_{-}\right)$. Given any finite dimensional left $U_{q}(\mathfrak{p})$ module $V$, we form the tensor product $V \otimes_{\mathbb{C}} G_{q}$, which is a subspace of functions $U_{q}(\mathfrak{g}) \rightarrow V:$

$$
\begin{aligned}
\zeta & =\sum v_{i} \otimes f_{i} \in V \otimes G_{q}, \\
x & \in U_{q}(\mathfrak{g}), \\
\zeta(x) & =\sum f_{i}(x) v_{i} .
\end{aligned}
$$

The left actions '.' and 'o' of $U_{q}(\mathfrak{g})$ on $G_{q}$ can be extended in an obvious way to actions on $V \otimes_{\mathbb{C}} G_{q}$

$$
\begin{aligned}
x \cdot \zeta & =\sum(-1)^{[x]\left[v_{i}\right]} v_{i} \otimes x \cdot f_{i}, \\
x \circ \zeta & =\sum(-1)^{[x]\left[v_{i}\right]} v_{i} \otimes x \circ f_{i}, \quad x \in U_{q}(\mathfrak{g}) .
\end{aligned}
$$

Furthermore, there also exists a co - action $\omega$ of $G_{q}$ on $V \otimes_{\mathbb{C}} G_{q}$ defined by $\omega=i d_{V} \otimes \Delta^{\prime}$, where $\Delta^{\prime}$ represents the opposite co - multiplication of $G_{q}$.

Consider the subspace of $V \otimes_{\mathbb{C}} G_{q}$ defined by

$$
\mathcal{O}^{V}=\left\{\zeta \in V \otimes_{\mathbb{C}} G_{q} \mid p \circ \zeta=\left(S(p) \otimes i d_{G_{q}}\right) \zeta, \forall p \in U_{q}(\mathfrak{p})\right\}
$$

Lemma $3 \mathcal{O}^{V}$ furnishes a left $U_{q}(\mathfrak{g})$ module under '.', and at the same time a right $G_{q}$ co - module under $\omega$. 
Proof: The Lemma can be confirmed by direct calculations. For $x \in U_{q}(\mathfrak{g}), p \in U_{q}(\mathfrak{p})$, $\zeta \in \mathcal{O}^{V}$, we have

$$
\begin{aligned}
p \circ(x \cdot \zeta) & =(-1)^{[x][p]} x \cdot(p \circ \zeta) \\
& =\left(S(p) \otimes i d_{G_{q}}\right)(x \cdot \zeta) ; \\
\left(p \circ \otimes i d_{G_{q}}\right) \omega(\zeta) & =\left(p \circ \otimes i d_{G_{q}}\right)\left(i d_{V} \otimes \Delta^{\prime}\right) \zeta \\
& =\left(i d_{V} \otimes \tau\right)\left(i d_{V} \otimes i d_{G_{q}} \otimes p \circ\right)\left(i d_{V} \otimes \Delta\right) \zeta \\
& =\left(i d_{V} \otimes \tau\right)\left(i d_{V} \otimes \Delta^{\prime}\right)(p \circ \zeta) \\
& =\omega\left(S(p) \otimes i d_{G_{q}}\right) \zeta,
\end{aligned}
$$

where $\tau$ is the flip mapping.

We call $\mathcal{O}^{V}$ the induced $U_{q}(\mathfrak{g})$ module, and also the induced $G_{q}$ co - module, which gives rise to a representation of $G_{q}$. A conceptual understanding of $\mathcal{O}^{V}$ can be gained by considering its classical analog. Let $P$ be a parabolic subgroup of the complex Lie supergroup $S L(m \mid n)$, and $E$ a finite dimensional representation of $P$. Then $S L(m \mid n) \times_{P} E$, the quotient space of $S L(m \mid n) \times E$ under the equivalence relation $(g, v) \sim\left(g p, p^{-1} v\right)$ for all $p \in P$, defines a super vector bundle over the supermanifold $S L(m \mid n) / P$. A function $f: S L(m \mid n) \rightarrow E$ satisfying $f(g p)=p^{-1} f(g), \forall p \in P$ defines a section of the bundle $s_{f}: S L(m \mid n) / P \rightarrow S L(m \mid n) \times_{P} E$. Analogously, we may regard $\mathcal{O}^{V}$ as the vector space of sections of a quantum super vector bundle over the quantum counter part of $S L(m \mid n) / P$.

It is of great importance to systematically develop the theory of quantum homogeneous super vector bundles, and the subject will be investigated to depth in a forthcoming publication. In this paper, we will restrict ourselves to issues directly related to representation theory, and will not further ponder on noncommutative geometry, except for the last section, where we will discuss in some detail quantum projective superspaces when dealing with explicit realizations of the skew supersymmetric tensor irreps and their duals.

We have the following quantum analog of Frobenius reciprocity.

Proposition 5 Let $W$ be a quotient $U_{q}(\mathfrak{g})$ module of $\oplus_{k, l=0}^{\infty} \mathbb{E}^{\otimes k} \otimes\left(\mathbb{E}^{*}\right)^{\otimes l}$ ( the restriction of which furnishes a $U_{q}(\mathfrak{p})$ module in a natural way.). Then there is a canonical isomorphism

$$
\operatorname{Hom}_{U_{q}(\mathfrak{g})}\left(W, \mathcal{O}^{V}\right) \cong \operatorname{Hom}_{U_{q}(\mathfrak{p})}(W, V),
$$

Proof: We prove the Proposition by explicitly constructing the isomorphism, which we claim to be the linear map

$$
\begin{aligned}
F: \operatorname{Hom}_{U_{q}(\mathfrak{g})}\left(W, \mathcal{O}^{V}\right) & \rightarrow \operatorname{Hom}_{U_{q}(\mathfrak{p})}(W, V), \\
\psi & \mapsto \psi\left(1_{U_{q}(\mathfrak{g})}\right),
\end{aligned}
$$

with the inverse map

$$
\begin{aligned}
\bar{F}: \operatorname{Hom}_{U_{q}(\mathfrak{p})}(W, V) & \rightarrow \operatorname{Hom}_{U_{q}(\mathfrak{g})}\left(W, \mathcal{O}^{V}\right), \\
\phi & \mapsto \bar{\phi}
\end{aligned}
$$

where $\bar{\phi}$ is defined by

$$
\bar{\phi}(w)(x)=(-1)^{[x]([w]+1)} \phi(S(x) w), \quad x \in U_{q}(\mathfrak{g}), w \in W .
$$


As for $F$, we need to show that its image is contained in $\operatorname{Hom}_{U_{q}(\mathfrak{p})}(W, V)$. This is indeed the case, as

$$
\begin{aligned}
p(F \psi(w)) & =(p \cdot \psi(w))\left(1_{U_{q}(\mathfrak{g})}\right) \\
& =(-1)^{[\psi][p]} F \psi(p w), \quad p \in U_{q}(\mathfrak{p}), \quad w \in W .
\end{aligned}
$$

In order to show that $\bar{F}$ is the inverse of $F$, we first need to demonstrate that the image $\operatorname{Im}(\bar{F})$ of $\bar{F}$ is contained in $\operatorname{Hom}_{U_{q}(\mathfrak{g})}\left(W, \mathcal{O}^{V}\right)$. Note that $\operatorname{Im}(\bar{F}) \subset$ $\operatorname{Hom}_{\mathbb{C}}\left(W, V \otimes G_{q}\right)$, since $W$ is a subquotient of $\bigoplus_{k, l=0}^{\infty} \mathbb{E}^{\otimes k} \otimes\left(\mathbb{E}^{*}\right)^{\otimes l}$. Some relatively simple manipulations lead to

$$
\begin{aligned}
(y \cdot \bar{\phi}(w))(x) & =(-1)^{[y][\bar{\phi}]+[x]([w]+[x]+[y])} \phi(S(x) y w) \\
& =(-1)^{[y][\bar{\phi}]} \bar{\phi}(y w)(x), \\
(p \circ \bar{\phi}(w))(x) & =(-1)^{[x]([w]+1)+[p][\phi]} \phi(S(p) S(x) w) \\
& =S(p)(\bar{\phi}(w)(x)), \quad x, y \in U_{q}(\mathfrak{g}), \quad p \in U_{q}(\mathfrak{p}), \quad w \in W .
\end{aligned}
$$

Therefore, $\operatorname{Im}(\bar{F}) \subset \operatorname{Hom}_{U_{q}(\mathfrak{g})}\left(W, \mathcal{O}^{V}\right)$.

Now we show that $F$ and $\bar{F}$ are inverse to each other. For $\psi \in \operatorname{Hom}_{U_{q}(\mathfrak{g})}\left(W, \mathcal{O}^{V}\right)$, and $\phi \in \operatorname{Hom}_{U_{q}(\mathfrak{p})}(W, V)$, we have

$$
\begin{aligned}
(F \bar{F} \phi)(w) & =(\bar{F} \phi)(w)\left(1_{U_{q}(\mathfrak{g})}\right) \\
& =\phi(w) \\
(\bar{F} F \psi)(w)(x) & =(-1)^{[x]([w]+1)}(F \psi)(S(x) w) \\
& =(-1)^{[x]([w]+1)} \psi(S(x) w)\left(1_{U_{q}(\mathfrak{g})}\right) \\
& =(-1)^{[x]([\psi(w)]+1)}(S(x) \cdot \psi(w))\left(1_{U_{q}(\mathfrak{g})}\right) \\
& =\psi(w)(x), \quad x \in U_{q}(\mathfrak{g}), \quad w \in W .
\end{aligned}
$$

This completes the proof of the Proposition.

4.3 Quantum Borel - Weil theorem for covariant and contravariant tensor irreps

In this subsection we study in detail the covariant and contravariant tensor irreps of $U_{q}(\mathfrak{g})$ within the framework of parabolic induction. Our main result here will be a quantum version of the Borel - Weil theorem for these irreps.

Let $V$ be a finite dimensional irreducible $U_{q}(\mathfrak{p})$ module, with the $U_{q}(\mathfrak{l})$ highest weight $\mu$ and $U_{q}(\mathfrak{l})$ lowest weight $\tilde{\mu}$. For the purpose of studying the tensor representations, we only need to consider

$$
\begin{aligned}
& \overline{\mathcal{O}}(\mu)=\mathcal{O}^{V} \cap\left(V \otimes G_{q}^{\pi}\right), \\
& \mathcal{O}(\mu)=\mathcal{O}^{V} \cap\left(V \otimes G_{q}^{\bar{\pi}}\right) .
\end{aligned}
$$

Let us study $\mathcal{O}(\mu)$ first. A typical element of $\mathcal{O}(\mu)$ is of the form

$$
\zeta=\sum_{\lambda \in \Lambda^{(1)}} \sum_{\alpha, \beta, i} c_{\alpha \beta, i}^{\lambda} v_{i} \otimes \bar{t}_{\alpha \beta}^{\left(\lambda^{\dagger}\right)}
$$


where $\left\{v_{i}\right\}$ is a basis of $V$, and the $c_{\alpha \beta, i}^{\lambda}$ are complex numbers. The $\bar{t}_{\alpha \beta}^{\left(\lambda^{\dagger}\right)}$ are elements of the Peter - Weyl basis for $G_{q}^{\bar{\pi}}$, which, needless to say, are polynomials in $\bar{t}_{a b}, a, b \in \mathbf{I}$. The property that $(p \circ \zeta)=\left(S(p) \otimes i d_{G_{q}}\right) \zeta, \forall p \in U_{q}(\mathfrak{p})$ leads to

$$
\sum_{\gamma, i}(-1)^{[p]\left([\gamma]+\left[v_{i}\right]\right)} c_{\alpha \gamma, i}^{\lambda} t_{\gamma \beta}^{(\lambda)}(p) v_{i}=\sum_{i} c_{\alpha \beta, i}^{\lambda} p v_{i}, \quad \forall p \in U_{q}(\mathfrak{p}) .
$$

Let $W(\lambda)$ with the basis $\left\{w_{\alpha}\right\}$ be the irreducible $U_{q}(\mathfrak{g})$ module associated with the irrep $t^{(\lambda)}$. We define the linear maps between $\mathbb{Z}_{2}$ graded vector spaces

$$
\begin{aligned}
\phi_{\lambda}^{(\alpha)}: W(\lambda) & \rightarrow V, \\
w_{\beta} & \mapsto \sum_{i} c_{\alpha \beta, i}^{\lambda} v_{i} .
\end{aligned}
$$

There is no particular significance attached to the maps at this stage, apart from the mere fact that they can be employed to re - express equation (18) as

$$
\sum_{\gamma}(-1)^{[p]\left[\phi_{\lambda}^{(\alpha)}\right]} t_{\gamma \beta}^{(\lambda)}(p) \phi_{\lambda}^{(\alpha)}\left(w_{\gamma}\right)=p \phi_{\lambda}^{(\alpha)}\left(w_{\beta}\right) .
$$

We emphasize that this equation is entirely equivalent to (18). Now something of crucial importance appears: this equation requires that each $\phi_{\lambda}^{(\alpha)}$ be a $U_{q}(\mathfrak{p})$ module homomorphism of degree $\left[\phi_{\lambda}^{(\alpha)}\right]$. Lemma 2 forces

$$
\phi_{\lambda}^{(\alpha)}=c_{\alpha} \phi_{\lambda}, \quad c_{\alpha} \in \mathbb{C},
$$

and $\phi_{\lambda}$ may be nonzero only when

$$
\begin{aligned}
& \text { i). } \quad \bar{\lambda}=\tilde{\mu}, \quad \text { if } U_{q}(\mathfrak{p})=U_{q}\left(\mathfrak{p}_{+}\right), \\
& \text {ii). } \quad \lambda=\mu \text {, if } U_{q}(\mathfrak{p})=U_{q}\left(\mathfrak{p}_{-}\right) \text {. }
\end{aligned}
$$

In these cases, $\mathcal{O}(\mu)$ is spanned by

$$
\zeta_{\alpha}=\sum_{\beta} \phi_{\lambda}\left(w_{\beta}\right) \otimes \bar{t}_{\alpha \beta}^{\left(\lambda^{\dagger}\right)}
$$

which are obviously linearly independent. Furthermore,

$$
x \cdot \zeta_{\alpha}=(-1)^{[x]\left[\phi_{\lambda}\right]} \sum_{\beta} t_{\beta \alpha}^{(\lambda)}(x) \zeta_{\beta}, \quad x \in U_{q}(\mathfrak{g}) .
$$

The case of $\overline{\mathcal{O}}(\mu)$ can be studied in exactly the same way. To summarize, we have the following quantum analog of Borel - Weil theorem for the covariant and contravariant tensor irreps

Proposition 6 As $U_{q}(\mathfrak{g})$ modules,

$$
\begin{aligned}
& \mathcal{O}(\mu) \cong\left\{\begin{array}{lll}
W\left((-\tilde{\mu})^{\dagger}\right), & \text { if } \tilde{\mu} \in-\Lambda^{(2)}, & U_{q}(\mathfrak{p})=U_{q}\left(\mathfrak{p}_{+}\right), \\
W(\mu), & \text { if } \mu \in \Lambda^{(1)}, & U_{q}(\mathfrak{p})=U_{q}\left(\mathfrak{p}_{-}\right), \\
\{0\}, & \text { otherwise. }
\end{array}\right. \\
& \overline{\mathcal{O}}(\mu) \cong\left\{\begin{array}{lll}
W\left((-\tilde{\mu})^{\dagger}\right), & \text { if } \tilde{\mu} \in-\Lambda^{(1)}, & U_{q}(\mathfrak{p})=U_{q}\left(\mathfrak{p}_{+}\right), \\
W(\mu), & \text { if } \mu \in \Lambda^{(2)}, & U_{q}(\mathfrak{p})=U_{q}\left(\mathfrak{p}_{-}\right), \\
\{0\}, & \text { otherwise. }
\end{array}\right.
\end{aligned}
$$

In the Proposition, the notation $W(\lambda)$ signifies the irreducible $U_{q}(\mathfrak{g})$ module with highest weight $\lambda$. 


\section{QUANTUM PROJECTIVE SUPERSPACES, SKEW SUPERSYMMETRIC TENSOR IRREPS AND THEIR DUALS}

We will apply the general theory developed in the last section to study two infinite classes of irreps, namely, the skew supersymmetric tensor irreps and their dual irreps. Explicit realizations of these irreps will be given in terms of sections of quantum super vector bundles over quantum projective superspaces.

\subsection{Quantum projective superspaces}

Let $U_{q}\left(\mathfrak{g}^{\prime}\right), \mathfrak{g}^{\prime}=g l(m \mid n-1)$, be the subalgebra of $U_{q}(\mathfrak{g})$ generated by the following elements

$$
\left\{K_{a}, a \in \mathbf{I}^{\prime} ; E_{c c+1}, E_{c+1}, \quad c \in \mathbf{I}^{\prime} \backslash\{m+n-1\}\right\} .
$$

Clearly $U_{q}\left(\mathfrak{g}^{\prime}\right)$ is a Hopf subalgebra. Define

$$
\begin{aligned}
& \mathcal{A}_{+}=\left\{f \in G_{q}^{\pi} \mid f(x p)=\epsilon(p) f(x), \forall x \in U_{q}(\mathfrak{g}), p \in U_{q}\left(\mathfrak{g}^{\prime}\right)\right\}, \\
& \mathcal{A}_{-}=\left\{f \in G_{q}^{\pi} \mid f(x p)=\epsilon(p) f(x), \forall x \in U_{q}(\mathfrak{g}), p \in U_{q}\left(\mathfrak{g}^{\prime}\right)\right\} .
\end{aligned}
$$

The Hopf algebra structure of $U_{q}\left(\mathfrak{g}^{\prime}\right)$ implies that both $\mathcal{A}_{+}$and $\mathcal{A}_{-}$are subalgebras of $G_{q}$. Together they generate another subalgebra of $G_{q}$, which we will denote by $\mathbb{S}_{q}^{m \mid n-1}$. Set

$$
z_{a}=t_{a m+n}, \quad \bar{z}_{a}=\bar{t}_{a m+n}, \quad a \in \mathbf{I} .
$$

Then $z_{a}$ and $\bar{z}_{a}$ are conjugate to each other under the $*$ - operation with $\theta=0$. More explicitly,

$$
*\left(z_{a}\right)=\bar{z}_{a}, \quad \forall a \in \mathbf{I} .
$$

Now $\mathbb{S}_{q}^{m \mid n-1}$ is generated by the $z$ 's and $\bar{z}$ 's, which satisfy the following commutations relations

$$
\begin{aligned}
z_{a} z_{b} & =(-1)^{\left[z_{a}\right]\left[z_{b}\right]} q z_{b} z_{a}, \quad a<b, \\
\left(z_{c}\right)^{2} & =0, \quad c \leq m ; \\
\bar{z}_{a} \bar{z}_{b} & =(-1)^{\left[\bar{z}_{a}\right]\left[\bar{z}_{b}\right]} q^{-1} \bar{z}_{b} \bar{z}_{a}, \quad a<b, \\
\left(\bar{z}_{c}\right)^{2} & =0, \quad c \leq m ; \\
\bar{z}_{a} z_{b} & =q(-1)^{\left[\bar{z}_{a}\right]\left[z_{b}\right]} z_{b} \bar{z}_{a}+\delta_{a b}\left\{\left(1-q_{a}^{-1}\right) \bar{z}_{a} z_{a}\right. \\
& \left.-(-1)^{\left[\bar{z}_{a}\right]}\left(q-q^{-1}\right) \sum_{c<a} \bar{z}_{c} z_{c}\right\}, \quad \forall a, b \in \mathbf{I}, \\
\sum_{c \in \mathbf{I}} \bar{z}_{c} z_{c} & =1 .
\end{aligned}
$$

It can be shown that the last two equations imply that

$$
\sum_{c \in \mathbf{I}} q^{\left(2 \rho, \epsilon_{c}\right)} z_{c} \bar{z}_{c}=q^{\left(2 \rho, \epsilon_{m+n}\right)} .
$$


$\mathbb{S}_{q}^{m \mid n-1}$ furnishes a right $G_{q}$ co - module algebra, with the co - module action $\omega: \mathbb{S}_{q}^{m \mid n-1}$ $\rightarrow \mathbb{S}_{q}^{m \mid n-1} \otimes G_{q}$ defined by

$$
\begin{aligned}
\omega\left(z_{a}\right) & =\sum_{c \in \mathbf{I}} z_{c} \otimes t_{a c}, \\
\omega\left(\bar{z}_{a}\right) & =\sum_{c \in \mathbf{I}} \bar{z}_{c} \otimes \bar{t}_{a c} .
\end{aligned}
$$

Also, $\mathbb{S}_{q}^{m \mid n-1}$ gives rise to a right $U_{q}(\mathfrak{g})$ module algebra with the module action ' $\circ$ ' defined by (16). This module algebra structure restricts naturally to a module algebra structure over $U_{q}\left(\mathfrak{g}^{\prime}\right) \otimes U_{q}(g l(1))$, where $U_{q}(g l(1))$ is generated by $K_{m+n}^{ \pm 1}$. The action of $U_{q}\left(\mathfrak{g}^{\prime}\right)$ on $\mathbb{S}_{q}^{m \mid n-1}$ is trivial as following the definitions of $\mathcal{A}_{ \pm} ; U_{q}(g l(1))$ also acts in a very simple manner. To be explicit, we introduce the notations that for $L=\left(\theta_{1}, \ldots, \theta_{m} ; l_{1}, \ldots, l_{n}\right) \in\{0,1\}^{\otimes m} \otimes \mathbb{Z}_{+}^{\otimes n},|L|=\sum_{i=1}^{m} \theta_{i}+\sum_{\mu=1}^{n} l_{\mu}$. Set

$$
\begin{aligned}
Z^{L} & =z_{1}^{\theta_{1}} \ldots z_{m}^{\theta_{m}} z_{m+1}^{l_{1}} \ldots z_{m+n}^{l_{n}}, \\
\bar{Z}^{L} & =\bar{z}_{1}^{\theta_{1}} \ldots \bar{z}_{m}^{\theta_{m}} \bar{z}_{m+1}^{l_{1}} \ldots \bar{z}_{m+n}^{l_{n}} .
\end{aligned}
$$

Then for any $k \in \mathbb{Z}$, and $p \in U_{q}\left(\mathfrak{g}^{\prime}\right)$, we have

$$
\left(p K_{m+n}^{k}\right) \circ\left(Z^{L} \bar{Z}^{L^{\prime}}\right)=\epsilon(p) q^{k\left(\left|L^{\prime}\right|-|L|\right)} Z^{L} \bar{Z}^{L^{\prime}} .
$$

We will define the quantum projective superspace $\mathbb{C} P_{q}^{m \mid n-1}$ to be the $U_{q}(g l(1))$ invariant subalgebra of $\mathbb{S}_{q}^{m \mid n-1}$, namely,

$$
\mathbb{C} P_{q}^{m \mid n-1}=\left(\mathbb{S}_{q}^{m \mid n-1}\right)^{U_{q}(g l(1))} .
$$

\subsection{Skew supersymmetric tensor irreps and their duals}

We specialize $U_{q}\left(\mathfrak{p}_{+}\right)$and $U_{q}\left(\mathfrak{p}_{-}\right)$to the case with $\Theta=\mathbf{I}^{\prime} \backslash\{m+n-1\}$. Consider a one - dimensional irreducible $U_{q}\left(\mathfrak{p}_{+}\right)$module $V_{+}=\mathbb{C} v$ such that

$$
\begin{aligned}
E_{b b+1} v= & E_{c+1 c} v=0, \\
K_{b} v= & v, \\
K_{m+n} v= & q^{-k} v, \\
k \in \mathbb{Z}_{+}, & b, c \in \mathbf{I}^{\prime}, c<m+n-1,
\end{aligned}
$$

and denote the associated representation by $\phi$. Define

$$
\overline{\mathcal{O}}_{k}=\left\{\zeta \in V_{+} \otimes G_{q}^{\pi} \mid(p \circ \zeta)(x)=\phi(S(p)) \zeta(x), \forall x \in U_{q}(\mathfrak{g}), p \in U_{q}\left(\mathfrak{p}_{+}\right)\right\} .
$$

Direct calculations can show that

$$
\overline{\mathcal{O}}_{k}=\bigoplus_{|L|=k} \mathbb{C} v \otimes Z^{L}
$$

where $Z^{L}$ is defined by (22). Then $\overline{\mathcal{O}}_{k}$ gives rise to the rank $k$ skew supersymmetric tensor irrep of $U_{q}(\mathfrak{g})$, with the highest weight

$$
\lambda= \begin{cases}\sum_{i=1}^{k} \epsilon_{i}, & k \leq m \\ \sum_{i=1}^{m} \epsilon_{i}+(k-m) \epsilon_{m+1}, & k>m\end{cases}
$$


Now let $V_{-}=\mathbb{C} w$ be a one dimensional irreducible $U_{q}\left(\mathfrak{p}_{-}\right)$module such that

$$
\begin{aligned}
E_{c c+1} v= & E_{b+1 b} v=0, \\
K_{b} v= & v \\
K_{m+n} v= & q^{k} v \\
k \in \mathbb{Z}_{+}, & b, c \in \mathbf{I}^{\prime}, c<m+n-1,
\end{aligned}
$$

and denote the corresponding irrep by $\psi$. Define

$$
\mathcal{O}_{k}=\left\{\zeta \in V_{-} \otimes G_{q}^{\bar{\pi}} \mid(p \circ \zeta)(x)=\psi(S(p)) \zeta(x), \forall x \in U_{q}(\mathfrak{g}), p \in U_{q}\left(\mathfrak{p}_{-}\right)\right\} .
$$

Then

$$
\mathcal{O}_{k}=\bigoplus_{|L|=k} \mathbb{C} w \otimes \bar{Z}^{L}
$$

This time $\mathcal{O}_{k}$ yields an irrep with highest weight

$$
\lambda=-k \epsilon_{m+n},
$$

which is dual to the rank $k$ skew supersymmetric tensor irrep.

\section{References}

[1] A.J. Bracken, M.D. Gould and R.B. Zhang, Quantum supergroups and solutions of the Yang - Baxter equation, Mod Phys Lett A5(1990) 831 - 840;

R.B. Zhang, A. J. Bracken and M. D. Gould, Solution of the graded Yang - Baxter equation associated with the vector representation of $U_{q}(\operatorname{osp}(M / 2 n))$, Phys Lett B257 (1991) 133 - 139.

[2] M. Chaichian and P.P. Kulish, Phys Lett B234 (1990) 72;

R. Floreanini, V. P. Spiridonov and L. Vinet, Commun Math Phys 137 (1991) 149;

M. Scheunert, Serre - type relations for special linear Lie superalgebras, Lett Math Phys 34 (1993) 320;

H. Yamane, A Serre type theorem for affine Lie superalgebras and their quantized universal enveloping superalgebras, Proc Japan Acad bf A70 (1994) 31.

[3] V. G. Drinfeld, Quantum groups, Proc. Internl. Cong. Math., Berkeley, 1 (1986) 789; M. Jimbo, $A q$ - difference analog of $U(g)$ and the Yang - Baxter equation, Lett. math. Phys., 10 (1985) 63.

[4] J. H. H. Perk and C. L. Schultz, Phys. Lett. A84 (1981) 407;

V. V. Bazhanov and A. G. Shadrikov, Theor. Math. Phys. 73 (1987) 1302.

[5] M. D. Gould, R. B. Zhang and A. J. Bracken, Quantum double construction for graded Hopf algebras, Bull Australian Math Soc 47 (1993) 353 - 375;

$\mathrm{H}$. Yamane, Universal $R$ - matrices for quantum groups associated to Lie superalgebras, Proc. Japan. Acad. bf A67 (1991) 108;

S. M. Khoroshkin and V. N. Tolstoy, Universal $R$ - matrix for quantized (super)algebras, Commun. Math. Phys. 141 (1991) 599. 
[6] R. B. Zhang, Finite dimensional irreducible representations of the quantum supergroup $U_{q}(g l(m / n))$, J. Math. Phys., 34(1993) 1236 - 1254.

[7] R. B. Zhang, Finite dimensional representations of $U_{q}(C(n))$ at arbitrary $q$, J Phys A26 (1993) 7041 - 7059;

T. D. Palev, N. I. Stoilova and J. Van der Jeugt, Finite - dimensional representations of the quantum superalgebra $U_{q}[g l(m \mid n)]$ and related $q$ - identities, Commun. Math. Phys. 166 (1994) 367;

R. B. Zhang, The $g l(M \mid N)$ super Yangian and its finite dimensional representations, Lett Math Phys 37 (1996) 419 - 434;

R. B. Zhang, Symmetrizable quantum affine superalgebras and their representations, J Math Phys, in press.

[8] A. J. Bracken, M. D. Gould, Y. Z. Zhang and G. W. Delius, Solutions to the quantum Yang-Baxter equation with extra non-additive parameters, J. Phys. 27 A, (1994) 65516561 ;

A. J. Bracken, M. D. Gould, J. R. Links and Y. Z. Zhang, A new supersymmetric and exactly solvable model of correlated electrons, Phys. Rev. Lett. 74 (1995) 2768-2771.

[9] R. B. Zhang, Braid group representations arising from quantum supergroups with arbitrary $q$ and link polynomials, J Math Phys 33 (1992) 3918 - 3930;

M. D. Gould, I. Tsohantjis and A. J. Bracken, Quantum supergroups and link polynomials, Rev. Math. Phys. 15 (1993) 533-549;

J. R. Links, M. D. Gould and R. B. Zhang, Quantum supergroups, link polynomials and representations of the braid generators, Rev. Math. Phys., 5 (1993) 345 - 361.

[10] R. B. Zhang, Quantum supergroups and topological invariants of three - manifolds, Rev Math Phys 7 (1995) 809 - 831;

R. B. Zhang and H. C. Lee, Lickorish invariant and quantum OSP(1|2), Mod Phys Lett $\mathbf{A}$, in press.

[11] S. L. Woronowicz, Compact matrix pseudo groups, Commun. Math. Phys. 111 (1987) 613 ;

L. D. Faddeev, N. Yu. Reshetikhin and L. A. Takhtajan, Quantization of Lie groups and Lie algebras, Leningrad Math. J. , 1 (1990) 193.

[12] Yu. I. Manin, Quantum Groups and Noncommutative Geometry, Universite de Montreal, Centre de Recherches Mathematiques, Montreal, PQ (1988);

S. L. Woronowicz, Differential calculus on compact matrix pseudo groups ( quantum groups ), Commun. Math. Phys. 122 (1989) 125;

C. S. Chu, P. M. Ho and B. Zumino, Some complex quantum manifolds and their geometry, Preprint.

[13] V. Lakshmibai and N. Yu. Reshetikhin, Quantum deformation of flag and Schubert schemes, C. R. Acad. Sci. Paris. Ser. I. Math. 313 No 3. (1991) 121 - 126.

[14] M. D. Gould and R. B. Zhang, Classification of all star irreps of $g l(m \mid n)$, J. Math. Phys., 31 (1990) 2552 - 2559.

[15] M.D. Gould and M. Scheunert, Classification of finite dimensional unitary irreps for $U_{q}[g l(m \mid n)]$, J. Math. Phys. 36 (1995) 435-452.

[16] S. L. Woronowicz, Tannaha - Krein duality for compact matrix pseudo groups, Inv. Math., 93 (1988) 35. 
[17] E. Hewitt and K. Ross, Abstract harmonic analysis, vol.2. , 2nd edition, Springer Verlag, New York (1979). 